PROCEEDINGS OF THE

AMERICAN MATHEMATICAL SOCIETY

Volume 126, Number 12, December 1998, Pages 3545-3547

S $0002-9939(98) 04620-6$

\title{
A NOTE ON INVARIANCE OF SPECTRUM FOR SYMMETRIC BANACH *-ALGEBRAS
}

\author{
BRUCE A. BARNES
}

(Communicated by David R. Larson)

\begin{abstract}
Let $A$ be a symmetric Banach *-algebra, let $B$ be a Banach algebra, and assume that $A \subseteq B$. A result is proved giving conditions which imply that every element of $A$ has the same spectrum in both $A$ and $B$.
\end{abstract}

\section{INTRODUCTION}

Let $A$ be a Banach algebra. The complete algebra norm on $A$ will be denoted \|\|$_{A}$. For $a \in A, \sigma_{A}(a)$ is the spectrum of $a$ in $A$. Now assume that, in addition, $A$ is a *-algebra. Then $A$ is symmetric if $\sigma_{A}\left(a^{*} a\right) \subseteq[0, \infty)$ for all $a \in A$. Basic information concerning symmetric *-algebras can be found in [4, Chapter IV, Section 7]. All $C^{*}$-algebras are symmetric [4, Theorem (4.6.9)]. Also, Shirali's Theorem [1, Theorem 5, p. 226] implies that a Banach *-algebra $A$ is symmetric if and only if $A$ is hermitian; meaning, $\sigma_{A}(a) \subseteq \mathbf{R}$ for all $a=a^{*} \in A$.

There has been recent interest in the question:

If $A$ is a $C^{*}$-algebra, and $\pi: A \rightarrow B(X)$ is an isomorphism of $A$ into the algebra of all bounded linear operators on a Banach space $X$, then when does it hold that $\sigma_{A}(a)=\sigma(\pi(a))$, the operator spectrum of $\pi(a)$, for all $a \in A$ ?

Some results on this question can be found in [3]. Here we prove a theorem concerning symmetric ${ }^{*}$-algebras which has some bearing on this question. A corollary of this theorem generalizes some results in [3].

\section{THE RESULTS}

Theorem. Let $A$ and $B$ be Banach algebras with $A$ a closed subalgebra of $B$. When $A$ has a unit, then assume that this element is also the unit of $B$. Further, assume that $A$ is a symmetric * -algebra with continuous involution such that either

(i) the embedding of $A$ in $B$ is continuous; or

(ii) $A$ is semsimple.

Then $\sigma_{A}(a)=\sigma_{B}(a)$ for all $a \in A$.

Proof. First note that in either case (i) or (ii), the norms of $A$ and $B$ are equivalent on $A$. For if (i) holds, then this follows from the Open Mapping Theorem, and when (ii) holds, this is a consequence of Johnson's Uniqueness of Norm Theorem.

Received by the editors April 11, 1997.

1991 Mathematics Subject Classification. Primary 46K99, 46L05.

Key words and phrases. Symmetric Banach ${ }^{*}$-algebra, invariance of spectrum, $C^{*}$-algebra.

(C)1998 American Mathematical Society 
Now assume that $B$ has a unit 1 , and $1 \in A$. Assume $a \in A$ with $a^{-1} \in B$. By symmetry, $\left(n^{-1}+a a^{*}\right)^{-1} \in A$ for all integers $n \geq 1$. Suppose $a a^{*}$ is not invertible in $A$. Then by a standard argument,

$$
b_{n}=\left(n^{-1}+a a^{*}\right)^{-1} /\left\|\left(n^{-1}+a a^{*}\right)^{-1}\right\|_{A}
$$

has the properties $b_{n}=b_{n}^{*} \in A,\left\|b_{n}\right\|_{A}=1$, and $\left\|a a^{*} b_{n}\right\|_{A} \rightarrow 0$ as $n \rightarrow \infty$. Since the norms on $A$ and $B$ are equivalent on $A$, and

$$
\left\|a^{*} b_{n}\right\|_{B}=\left\|a^{-1}\left(a a^{*} b_{n}\right)\right\|_{B} \leq\left\|a^{-1}\right\|_{B}\left\|a a^{*} b_{n}\right\|_{B} \rightarrow 0,
$$

we have $\left\|a^{*} b_{n}\right\|_{A} \rightarrow 0$. Thus, taking the involution, $\left\|b_{n} a\right\|_{A} \rightarrow 0$, so $\left\|b_{n} a\right\|_{B} \rightarrow 0$. Multiplying $\left\{b_{n} a\right\}$ on the right by $a^{-1}$ in $B$, we have $\left\|b_{n}\right\|_{B} \rightarrow 0$, and finally $\left\|b_{n}\right\|_{A} \rightarrow 0$, a contradiction. The contradiction implies that $a a^{*}$ is invertible in $A$. Set $c=\left(a a^{*}\right)^{-1} \in A$, so $a a^{*} c=1$, and therefore, $a^{-1}=a^{*} c \in A$.

In the case where $A$ does not have a unit, one can be adjoined in the usual way (adjoin the unit of $B$ if $B$ has a unit). The resulting unital *algebra $A_{1}$ is symmetric and a closed subalgebra of $B_{1}$ (or $B$ ). The result then follows from the argument above.

The corollary we now prove applies to the question in the Introduction.

Corollary 1. Let $A$ be a $C^{*}$-algebra. Assume $\pi: A \rightarrow B$ is a continuous algebra homomorphism of $A$ into a Banach algebra $B$. Set $J=\operatorname{ker}(\pi)$. If e is a unit for $A$ modulo $J$, then assume $\pi(e)$ is the unit for $B$. For all $a \in A$

$$
\sigma_{A / J}(a+J)=\sigma_{B}(\pi(a)) .
$$

Proof. By standard results which hold for $C^{*}$-algebras, $J$ is a closed *-ideal of $A, A / J$ is a $C^{*}$-algebra, and $A / J$ is symmetric. Also, by a result of S. Cleveland $[2$, Lemma 5.3], the image $\pi(A)$ is a closed subalgebra of $B$. Therefore the Theorem applies to prove the result.

The next corollary generalizes some results in [3].

Corollary 2. Let $A$ be a $C^{*}$-algebra which is a subalgebra of a Banach algebra $D$. Assume that there exists a constant $M>0$ such that $\|a\|_{D} \leq M\|a\|_{A}$ for all $a \in A$. Let $K$ be a closed subspace of $D$ with the property that for all $a \in A$ and $k \in K, a k \in K$. Let $\pi: A \rightarrow B(K)$ be defined by $\pi(a) k=a k, a \in A, k \in K$. Set $J=\operatorname{ker}(\pi)$. If e is a unit for $A$ modulo $J$, then assume ek $=k$ for all $k \in K$.

Then $\sigma_{A / J}(a+J)=\sigma_{B(K)}(\pi(a))$ for all $a \in A$.

Proof. For all $a \in A$ and $k \in K$,

$$
\|\pi(a) k\|_{D}=\|a k\|_{D} \leq\|a\|_{D}\|k\|_{D} \leq M\|a\|_{A}\|k\|_{D} .
$$

Therefore, $a \rightarrow \pi(a)$ is a continuous homomorphism of $A$ into $B(K)$. Thus, Corollary 1 implies the result.

There are some interesting applications of the Theorem to the representation theory of $L^{1}(G)$. These will be considered in a subsequent paper which is joint work of the present author with Professor Ajit I. Singh.

Bruce Barnes thanks the Mathematics Department of the University of Delhi for their hospitality during his visit there. 


\section{REFERENCES}

1. F. F. Bonsall and J. Duncan, Complete Normed Algebras, Springer-Verlag, Berlin, 1973. MR 54:11013

2. S. Cleveland, Homomorphisms of non-commutative *-algebras, Pacific J. Math. 13 (1963), 1097-1109. MR 28:1500

3. J. Daughtry, A. Lambert, and B. Weinstock, Invariance of spectrum for representations of $C^{*}$-algebras on Banach spaces, Proceedings of the AMS 125 (1997), 189-198. MR 97c:46067

4. C. Rickart, Banach Algebras, Van Nostrand, 1960.

Department of Mathematics, University of Oregon, Eugene, Oregon 97403

E-mail address: barnes@darkwing.uoregon.edu 